\title{
A new method for measuring the thermal conductivity of small insulating samples
}

\author{
Yves Jannot $^{1 *}$, Sébastien Schaefer ${ }^{2}$, Alain Degiovanni ${ }^{1,3}$, Jérémy Bianchin ${ }^{1}$, Vanessa Fierro ${ }^{2}$, \\ and Alain Celzard ${ }^{2}$ \\ ${ }^{1}$ Université de Lorraine, CNRS, LEMTA, F-54500 Vandœuvre-lès-Nancy, France \\ ${ }^{2}$ Université de Lorraine, CNRS, IJL, UMR 7198, F-88000, Epinal, France \\ ${ }^{3}$ Université Internationale de Rabat, Pôle Energie, LERMA, Rocade Rabat-Salé, 11100, Sala \\ Al Jadida, Morocco
}

\begin{abstract}
Due to the limited size of samples prepared at the lab scale, characterizing the thermal properties of new materials may be problematic, and this makes it all the more difficult to optimize them. This statement especially applies to the development of innovative thermal (super)insulators. In this work we demonstrate that the Hot Disk method, which might be preferred for such kind of studies because of the low sample size it can allow in practice, is unable to provide realistic values of thermal conductivity in the case of insulators. A new method was thus developed, called Calibrated Tiny Hot Plate (CTHP). The corresponding heat transfers were modelled, and the two unknowns of the model were obtained by calibration with insulating samples whose thermal conductivity was precisely measured with a reference (guarded hot plate) method. We show that the CTHP is able to estimate conductivities within the range $0.014-0.2 \mathrm{~W} \mathrm{~m}^{-1} \mathrm{~K}^{-1}$ based on samples having thicknesses ranging from 3 to $9 \mathrm{~mm}$ and diameters as low as $15 \mathrm{~mm}$. The accuracy was generally much better than $10 \%$, which is a remarkable result for characterizing so small insulating materials.
\end{abstract}

Keywords: Thermal conductivity; Small samples; Insulating materials; Measurement method.

\section{Introduction}

By definition, a material is a solid substance selected out of many others for its useful physical properties, allowing it to become an essential part of a system or an object. Materials characterization is thus a crucial task upon choosing a formulation, a synthesis method, or a production process. Depending on the kind of technology in which the considered material will be integrated, not all but a limited number of properties need to be precisely known. Among them, density on the one hand, and mechanical and thermal properties on the other hand, are often of paramount importance, whatever the final application. The first two characteristics can generally be easily measured on small samples, but this is rarely the case in practice for thermal properties, and especially as far as the conductivity of insulating materials is concerned.

\footnotetext{
"Corresponding author. Tel: + 333727443 08. E-mail address : yves.jannot@univlorraine.fr (Y. Jannot)
} 
Indeed, the heat transfer properties of poorly conducting materials are best measured with rather big samples. For instance, the guarded hot plate method ${ }^{1-3}$ requires typical sizes of $30 \times$ $30 \mathrm{~cm}^{2}$ according to the ISO 8302:1991 standard ${ }^{4}$. Such dimensions are problematic at the lab scale when new materials are developed. Most of the time, preparing new materials with improved properties or original structures is indeed costly and, in particular when new formulations and preparation methods are developed, upscaling the processes for producing large amounts is a true challenge. In some cases, the volume of the samples is also limited by the size of the reactors in which they are prepared, and this especially applies to aerogels. Aerogels are a special kind of materials prepared by sol-gel polymerization in a solvent, and whose porosity is produced by supercritical drying ${ }^{5-7}$. Such preparation step thus requires a special autoclave wherein the solvent saturating the porosity of the gels can be evacuated without collapse. As a result, extremely narrow pores are formed in which a Knudsen effect takes place, making these materials prone to present a thermal conductivity lower than air. These materials are thus called "superinsulators", with typical thermal conductivities within the range $0.012-0.025 \mathrm{~W} \mathrm{~m}^{-1} \mathrm{~K}^{-1}$.

Manufacturing large blocks of aerogels for determining their thermal conductivity by the guarded hot plate method is unrealistic for at least two reasons: (i) the capacity of the corresponding supercritical point driers should be extremely high, thus consuming tens or even hundreds $\mathrm{kg}$ of liquid $\mathrm{CO}_{2}$ for producing such large samples; and (ii) the time for such production would be tremendously long since the process is based on solvent exchange, the latter being driven by diffusion, whose characteristic time varies as the squared thickness. Therefore, not only making big monoliths is not possible at the lab scale, but optimizing this kind of materials also passes through the screening of many formulations. The corresponding syntheses and trials associated to such screening can only be achieved with small samples, which can be prepared rapidly, but whose thermal properties must be known before the best formulation can be up-scaled to the industrial level.

The same also applies, although to a lower extent, to insulating foams ${ }^{8}$. The latter fit into the class of traditional insulators, with typical conductivities within the range $0.030-0.050 \mathrm{~W} \mathrm{~m}^{-}$ ${ }^{1} \mathrm{~K}^{-1}$. The number of formulations that are required for finding a compromise between high mechanical strength and low thermal conductivity, which are two antagonistic properties (see ${ }^{9}$ for instance) require fast and accurate characterization, again based on small samples. The question of preparing and testing as much samples as possible, inevitably of limited dimensions, for selecting the most promising ones is especially important when it comes to the search of high-performance insulators for the renovation of buildings. In this context, superinsulators allow saving 3 to $5 \%$ of living area, compared to traditional insulators.

Methods exist for measuring the thermal conductivity of centimeter-sized samples, some of them being commercialized. This is the case of flash ${ }^{10,11}$, hot strip ${ }^{12,13}$, or hot disk ${ }^{14,15}$ methods, for instance. However, and whereas the corresponding suppliers claim that those methods are relevant for characterizing insulating materials, some intrinsic drawbacks induce huge errors on the measured values of thermal conductivity.

Thus, the flash method is intrinsically inappropriate to insulating samples for the following reasons ${ }^{16}$ : (i) insulators are often semi-transparent with respect to flash radiation; (ii) the 
measurement of surface temperature on a very light material is difficult to realize with precision; and (iii) the exchanges on the irradiated surface are often very different from the exchanges on the opposite surface (significant temperature differences). On the other hand, both hot strip and hot disk methods do not take into account the heat losses through the wires of the thermal sensor. It will be shown in this paper that the lower the sample size and the lower its thermal conductivity, the higher the contribution of these losses to the final result.

At the lab-scale, other methods have been developed, such as the centered hot plate ${ }^{17}$, based on samples of size $10 \times 10 \mathrm{~cm}^{2}$, or very recently the centered hot plate with convective guard $^{18}$, using samples as small as $4.5 \times 4.5 \mathrm{~cm}^{2}$. Whereas these methods present significant advances towards the thermal characterization of tiny samples, still they fail in determining accurately the conductivity of centimeter-size samples, since edge effects can never be suppressed at the center of materials having so small dimensions.

The purpose of the present work was thus to offer a new method allowing the thermal characterization of poorly conducting materials based on cylindrical samples of diameter 15 $\mathrm{mm}$, with an accuracy of $10 \%$. For that purpose, a steady-state method that we will call the Calibrated Tiny Hot Plate (CTHP) is offered here, having the following characteristics: (i) using a sensor whose heat capacity may be higher than that of the sample; (ii) based on the average surface temperature, given the impossibility of reaching a purely 1D transfer at the center of small samples; and (iii) in which the heat losses through the wires have been thoroughly calibrated. The dramatic impact of these losses, and hence the importance of such calibration, will be demonstrated with a standard measurement of thermal conductivity carried out with the Hot Disk method on an insulating sample of known conductivity. The new CTHP device will be described and tested with small samples belonging to the class of either traditional insulators of superinsulators, and having well-known conductivities formerly measured by the centered hot plate method with large samples.

\section{Principle and modeling of the CTHP method}

\subsection{Device}

The new CTHP device is described in figure 1 .

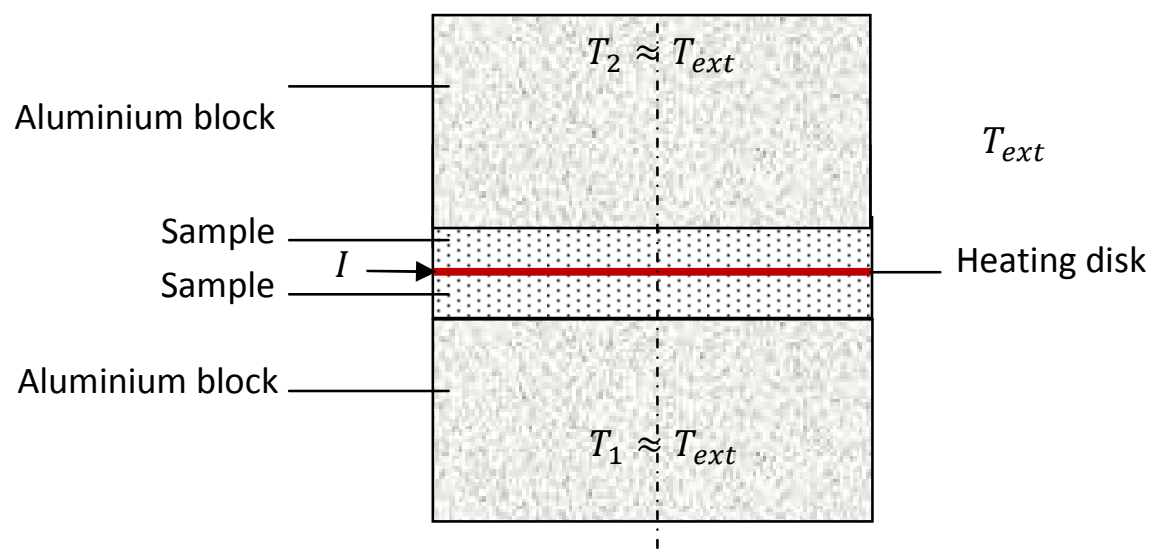

Figure 1. Block diagram of the experimental setup 
It consists of a thin heating disk of diameter $D$, inserted between two identical cylinders of the sample to be characterized having a given thickness $t$ but the same diameter $D$ as that of the thin heating disk. The faces of the latter are coated with metal, allowing maintaining a uniform temperature $T_{0}$ on those areas. The non-heated (thus external) sides of the cylinders are in close contact with aluminum blocks, also imposing uniform temperatures $T_{1}$ and $T_{2}$ on these areas. The whole device is placed in a thermally regulated enclosure at temperature $T_{\text {ext }}$. A current of intensity $I$ is forced to flow throughout the heating element of resistance $R_{e l}$, thereby producing a flux $R_{e l} I^{2}$ by Joule heating. Part of this heat flow rate is transferred through the samples whereas the rest, $\varphi_{\text {wires }}$, is dissipated towards the exterior through the connection pads of the current feed wires and through the thermocouple wires.

\subsection{Modeling}

Based on the setup described in Fig. 1, it can be assumed that:

- The samples to be characterized are isotropic;

- The loss coefficient $h$ (through convection and radiation) is the same for all measurements;

- The thermal resistances of the contacts are negligible with respect to those of the samples;

- The losses through the wires are proportional to the difference $T_{0}-T_{\text {ext }}$, and the proportionality factor is the same for all measurements.

Let:

$$
\varphi_{i}=f(\lambda, h, t)
$$

be the heat flow rate leaving the face at uniform temperature $T_{0}$ of the sample of thickness $t$ with the other face maintained at uniform temperature $T_{i}$ and the lateral face exchanging with the surrounding air at $T_{\text {ext }}$ with a global heat transfer coefficient $h$, as represented in Fig. 2. Here $\lambda$ is the thermal conductivity of the sample and $k$ is a coefficient characterizing the losses throughout the current feed wires and the thermocouple wires.

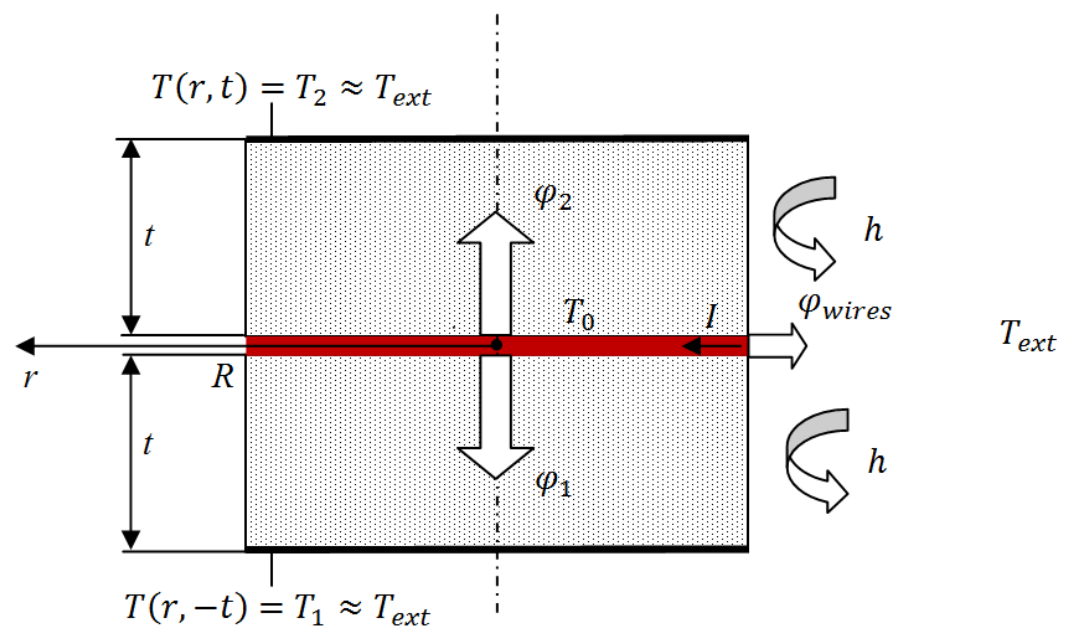

Figure 2. Schematization of the heat flows in the CTHP method

We set: $\theta_{i}=T_{i}-T_{\text {ext }}(i=1,2)$ and: $\theta_{0}=T_{0}-T_{\text {ext }}$ 
It was proved that ${ }^{19}: \theta_{i}=\sum_{n=0}^{\infty} \frac{\bar{\theta}_{l}(z) J_{0}\left(\frac{\omega_{n} r}{R}\right)}{N_{n}}$

with: $\bar{\theta}_{i}(z)=\frac{R^{2} J_{1}\left(\omega_{n}\right)}{\omega_{n}}\left\{\left[\theta_{i}-\theta_{0} \cosh \left(\frac{\omega_{n} t}{R}\right)\right] \frac{\sinh \left(\frac{\omega_{n} z}{R}\right)}{\sinh \left(\frac{\omega_{n} t}{R}\right)}+\theta_{0} \cosh \left(\frac{\omega_{n} z}{R}\right)\right\}$

and: $N_{n}=\frac{R^{2}}{2}\left[J_{0}^{2}\left(\omega_{n}\right)+J_{1}^{2}\left(\omega_{n}\right)\right]$

where $R$ is the radius of the sample, $J_{0}$ is the Bessel function of first kind and order 0 and $J_{1}$ is the Bessel function of first kind and order 1.

The flux density leaving the disk surface at temperature $T_{0}$ at a distance $r$ from its center can be calculated as:

$\phi_{i}(r)=-\lambda \frac{d \theta_{i}}{d z}(r, z=0)=-\lambda \sum_{n=0}^{\infty} \frac{J_{0}\left(\frac{\omega_{n} r}{R}\right)}{N_{n}} \frac{d \bar{\theta}_{i}}{d z}$

with: $\frac{d \bar{\theta}_{i}}{d z}(z=0)=\frac{R J_{1}\left(\omega_{n}\right)}{\sinh \left(\frac{\omega_{n} t}{R}\right)}\left[\theta_{i}-\theta_{0} \cosh \left(\frac{\omega_{n} t}{R}\right)\right]$

The value of the total heat flow rate leaving this surface can thus be deduced. It reads:

$\varphi_{i}=\int_{0}^{R} \phi_{i}(r) 2 \pi r d r=-\lambda \int_{0}^{R} \sum_{n=0}^{\infty} \frac{J_{0}\left(\frac{\omega_{n} r}{R}\right)}{N_{n}} \frac{d \bar{\theta}_{i}}{d z} 2 \pi r d r$

$\varphi_{i}=-\lambda \int_{0}^{R} \sum_{n=0}^{\infty} \frac{J_{0}\left(\frac{\omega_{n} r}{R}\right)}{N_{n}} \frac{R J_{1}\left(\omega_{n}\right)}{\sinh \left(\frac{\omega_{n} t}{R}\right)}\left[\theta_{i}-\theta_{0} \cosh \left(\frac{\omega_{n} t}{R}\right)\right] 2 \pi r d r$

$\varphi_{i}=-\lambda \sum_{n=0}^{\infty} \frac{R J_{1}\left(\omega_{n}\right)}{\sinh \left(\frac{\omega_{n} t}{R}\right)}\left[\theta_{i}-\theta_{0} \cosh \left(\frac{\omega_{n} t}{R}\right)\right] \int_{0}^{R} \frac{J_{0}\left(\frac{\omega_{n} r}{R}\right)}{N_{n}} 2 \pi r d r$

Now, since: $\int_{0}^{R} \frac{J_{0}\left(\frac{\omega_{n} r}{R}\right)}{N_{n}} 2 \pi r d r=2 \pi \frac{R^{2}}{\omega_{n}} J_{1}\left(\omega_{n}\right)$

we have : $\quad \varphi_{i}=-4 \pi R \lambda \sum_{n=0}^{\infty} \frac{J_{1}{ }^{2}\left(\omega_{n}\right)\left[\theta_{i}-\theta_{0} \cosh \left(\frac{\omega_{n} t}{R}\right)\right]}{\omega_{n} \sinh \left(\frac{\omega_{n} t}{R}\right)\left[J_{0}{ }^{2}\left(\omega_{n}\right)+J_{1}{ }^{2}\left(\omega_{n}\right)\right]}$

where $\omega_{n}$ is the solution of: $\omega_{n} J_{1}\left(\omega_{n}\right)=\frac{h R}{\lambda} J_{0}\left(\omega_{n}\right)$

The heat balance over the heating element reads: $R_{e l} I^{2}=\varphi_{1}+\varphi_{2}+\varphi_{\text {wires }}$

Finally, $\varphi_{\text {wires }}$ can be expressed as: $\varphi_{\text {wires }}=k\left(T_{0}-T_{\text {ext }}\right)$

The unknowns of the problem are thus $\lambda, k$ and $h$.

\subsection{Calibration}

The parameters $k$ and $h$ can be obtained by performing $M$ measurements $(M \geq 2)$ with samples of the same material of different thicknesses $t_{m}$ having a known thermal conductivity $\lambda_{\text {ref }}$. For each tested value of $h$, the value of $k$ is first determined so that the estimated value $\lambda_{\text {est }}$ of the thermal conductivity for the thinner sample is equal to the reference value $\lambda_{\text {ref }}$. Then, the thermal conductivity is estimated using the same values of $k$ and $h$ for the samples 
with higher thicknesses. The process is repeated until we find a value of $h$ such that the following sum is minimized:

$$
\sum_{m=1}^{M}\left[\lambda_{e s t_{m}}-\lambda_{r e f}\right]^{2}
$$

The device obviously has to ensure perfect reproducibility of the external conditions so as the assumption of constant values of $k$ and $h$ from one experiment to another still holds. Since $h$ may depend on the temperature, the calibration is only valid for one temperature.

\subsection{Measurement}

Once the values of $k$ and $h$ are determined, Eq. (13) in which:

- $\varphi_{1}$ and $\varphi_{2}$ are calculated from Eq. (11)

- $\varphi_{\text {wires }}$ is calculated from Eq. (14).

has one single unknown $\lambda$, and then can be numerically solved.

\section{Materials and methods}

\subsection{Materials}

Given that the two methods compared in the present study, i.e., the Hot Disk and the new method developed here, both use a sensor sandwiched between two identical samples (see below), the various tested materials were always considered in pairs. They were:

- 4 pairs of Styrodur ${ }^{\circledR}$ extruded polystyrene (XPS) foam samples of thicknesses 3.03, 4.46, 6.00 and $9.00 \mathrm{~mm}$

- 3 pairs of opacified expanded polystyrene (EPS) foam samples of thicknesses 2.95, 5.80 and $8.74 \mathrm{~mm}$

- 2 pairs of a superinsulating samples based on silica aerogel (SA) of thicknesses 2.90 and $4.94 \mathrm{~mm}$

- 1 pair of polyethylene (PE) foam samples of thickness $6.30 \mathrm{~mm}$

- 1 pair of polyvinyl chloride (PVC) samples of thickness $5.15 \mathrm{~mm}$.

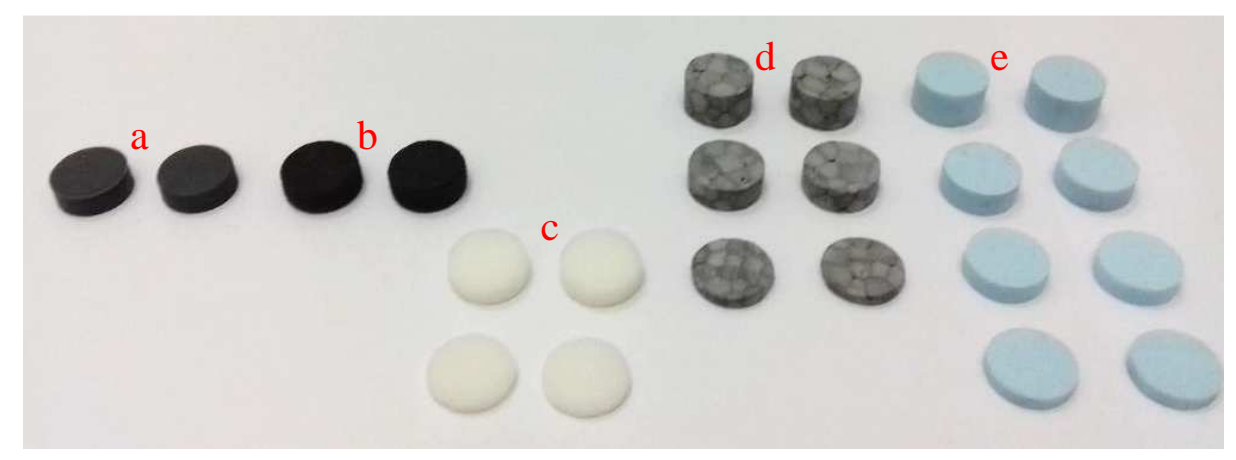

Figure 3. General view of all pairs of insulating samples studied in this work (see text): (a) PVC; (b) PE foam; (c) SA-based superinsulator; (d) opacified EPS foam; (e) XPS foam.

The thermal conductivity of each material was carefully measured at $25^{\circ} \mathrm{C}$ with the permanent centered hot plate method ${ }^{18}$, based on parallelepiped samples of section $100 \times 100 \mathrm{~mm}^{2}$. The 
corresponding values were considered as references for estimating the accuracy and the reliability of the two conductivity determination methods considered here.

All materials were then cut into cylindrical shape and gently polished using very fine sandpaper so that the final diameter was exactly $15 \mathrm{~mm}$. Fig. 3 shows the panel of sample pairs used in the present study.

\subsection{Methods}

\section{Hot Disk tests}

In order to evidence the limitations of the Hot Disk method applied to small insulating samples, the thermal conductivities of the aforementioned XPS foam and SA superinsulator (blue and white materials in Fig. 3, respectively) were measured by the transient plane source method with a commercial thermal conductivity analyzer (Hot Disk TPS 2500, ThermoConcept, France). The measurements were carried out at stable room temperature $\left(21^{\circ} \mathrm{C}\right)$ and relative humidity $(40 \% \mathrm{RH})$ using the 7577 probe (radius $2.001 \mathrm{~mm}$ ). The samples were in the form of two similar cylinders of diameter $D$ of at least $15.00 \mathrm{~mm}$, and of various thicknesses $t$. According to the Hot Disk manual, the optimal thickness must be in the range $10-40 \mathrm{~mm}$. However, due to scale-up limitations and to the way some lab-made materials are prepared, especially aerogels, most of samples present a thickness below $10 \mathrm{~mm}$. Thus, in spite of the aforementioned recommendation about thickness, samples thinner than $10 \mathrm{~mm}$, i.e., in the range $3-9 \mathrm{~mm}$, were tested with the Hot Disk method, with the aim of showing its limitations for small samples $(D \geq 15 \mathrm{~mm}$ and $t<10 \mathrm{~mm}$ ).

The Hot Disk sensor consists of an electrically conducting pattern in the shape of a double spiral, which has been etched out of a thin nickel foil and sandwiched between two thin sheets of Kapton ${ }^{\circledR}$. When the condition $t>3.75 \mathrm{~mm}$ (i.e., $t>D / 4$, according to the Hot Disk manual) is fulfilled, the maximum radius allowed for the probe is $r_{\max }=3.75 \mathrm{~mm}$. When $t<3.75 \mathrm{~mm}, r_{\max }$ is equal to $t$. Here, the minimum value of $t$ is $3 \mathrm{~mm}$. As a consequence, the 7577 probe (radius $2.001 \mathrm{~mm}$ ) is the largest and only probe that can be used for these experiments. The available penetration depth $\Delta P$ is equal to the minimal value between $t$ and $D / 2-r$ (where $r$ is the radius of the probe). Thus, if $t>5.5 \mathrm{~mm}$ is respected, the maximum value of $\Delta P$ is $D / 2-r$, i.e., $5.5 \mathrm{~mm}$. Otherwise $(t<5.5 \mathrm{~mm})$, the maximum of $\Delta P$ is equal to $t$.

The samples - sensor sandwich was then placed under an aluminum bell jar preserving it from convection and air turbulences. A light aluminum disk was also used to gently press together the surfaces of samples and probe and hence to maintain a thermal good contact. The aluminum disk was the same in all experiments and the applied stress was also constant. The device was left to equilibrate for 1 hour with the surroundings of the air-conditioned lab (a minimum of 20 min only is recommended for insulating materials according to the Hot Disk manual). From the temperature profile measured by one spiral after a heat pulse was delivered by the other spiral, the thermal conductivity was calculated by the Hot Disk 6.1 software. The characteristics of the heat pulse were also specified before starting the experiment. The selected duration of the pulse was in the $10-40 \mathrm{~s}$ range while the input power ranged from 3 
to $7 \mathrm{~mW}$. Only two combinations of power and pulse length were used: $10 \mathrm{~s}$ and $7 \mathrm{~mW}$, or 40 $\mathrm{s}$ and $3 \mathrm{~mW}$.

Such method is highly attractive because of the fast measurements and the limited sample size it allows in practice. However, some restrictions on the results must be respected. The total temperature increase during the heat pulse must be in the $3-7 \mathrm{~K}$ range while the temperature increase in the selected area of the graph used for the calculation must be in the $0.4-4 \mathrm{~K}$ range. The total characteristic time should take a value between 0.33 and $1 \mathrm{~s}$, and the mean deviation should be below $10^{-3} \mathrm{~K}$. Finally the penetration depth $\Delta P$ should respect the aforementioned criteria.

\section{Calibrated Tiny Hot Plate Device (CTHP)}

The CTHP device is based on a circular heater made of a metal foil sandwiched between two thin Kapton ${ }^{\circledR}$ sheets supplied by Captec (France). The two faces of the heater were covered with a $0.1 \mathrm{~mm}$-thin self-adhesive aluminum foil of identical area in order to homogenize the temperature on both sides. The outer dimensions of the heating element were $15 \mathrm{~mm}$ and 0.25 $\mathrm{mm}$ for diameter and thickness, respectively. A K-type thermocouple with a $0.05 \mathrm{~mm}$ diameter was next inserted between the heater and one aluminum foil, allowing measuring the temperature $T_{0}$, assumed to be uniform, of the heated faces of the samples.

Such heating element was then placed between two identical samples to be characterized, having fixed diameters of $15 \mathrm{~mm}$ and thicknesses $t$ already mentioned in section 3.1. The non-heated sides of the materials were maintained at temperatures $T_{1}$ and $T_{2}$, very close to the ambient temperature, $T_{\text {ext }}$, thanks to their close contact with two thermalized aluminum blocks, as shown in Fig. 4.

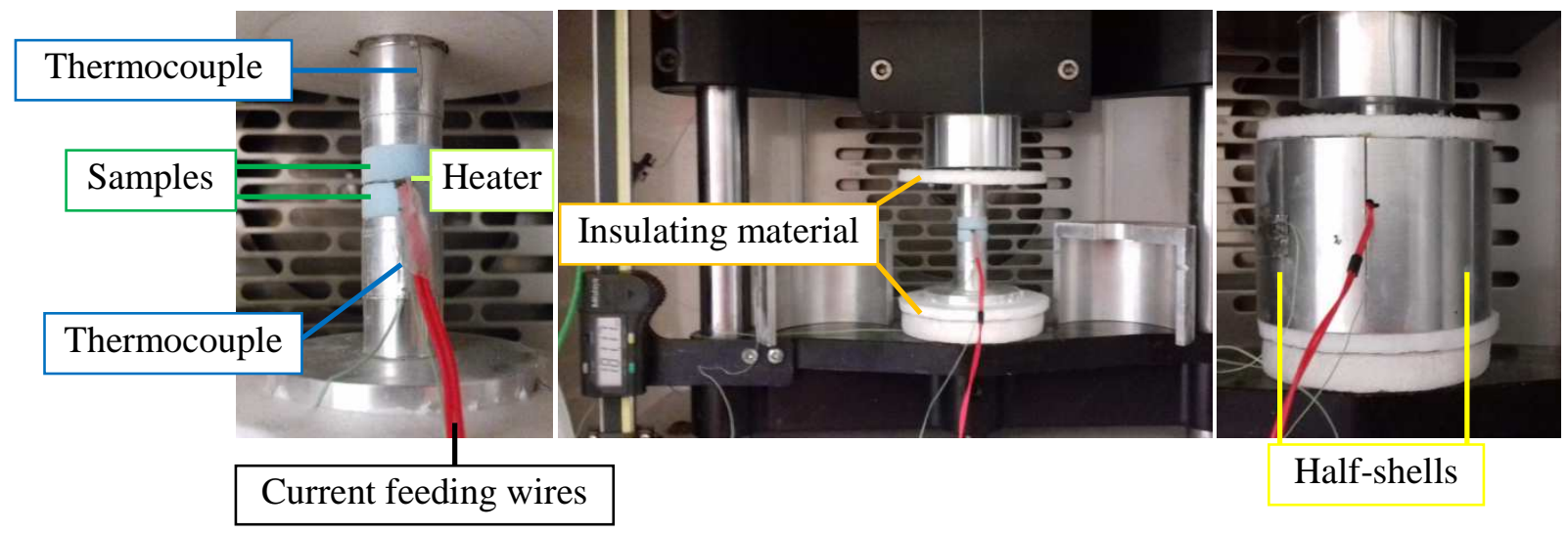

Figure 4. Side views of the lab-made device. From left to right: detail of the heater sandwiched between two identical samples (here XPS foam), themselves inserted between 2 aluminum blocks; general view of the setup gently compressed for allowing good thermal contacts; setup closed with half shells in the climate chamber.

The lateral sides were allowed to exchange heat with air by both convection and radiation with a global loss coefficient $h$. The device was surrounded laterally by two aluminum halfshells, and at top and bottom sides by two extruded polystyrene disks: (i) completely insulating it from outside air movements and thus ensuring the reproducibility of external 
conditions; and (ii) allowing an accurate measurement of $T_{\text {ext }}$ thanks to a thermocouple fixed on one half-shell. The whole device was finally placed in a climate chamber fixing the ambient temperature to $T_{\text {ext }}=20^{\circ} \mathrm{C}$.

\section{Results and discussion}

\subsection{Thermal conductivity of samples}

With the centered permanent hot plate ${ }^{18}$ used as a reference method, the thermal conductivity of each material was measured with an accuracy better than $3 \%$ and the corresponding results are given in Table 1. These values were used for checking the accuracy of the Hot Disk method on the one hand, and for calibrating the new method on the other hand.

Table 1. Thermal conductivity of all materials, measured by the centered permanent hot plate method at $25^{\circ} \mathrm{C}$ with samples of section $100 \times 100 \mathrm{~mm}^{2}$, and used as reference in the following.

\begin{tabular}{|l|l|}
\hline Sample & $\lambda_{\text {ref }}\left(\mathrm{W} \mathrm{m}^{-1} \mathrm{~K}^{-1}\right)$ \\
\hline XPS foam & 0.0308 \\
\hline EPS foam & 0.0324 \\
\hline SA superinsulator & 0.0141 \\
\hline PE foam & 0.0405 \\
\hline PVC & 0.193 \\
\hline
\end{tabular}

\subsection{Application of the Hot Disk method}

For the sake of comparison, the thermal conductivities of two materials were evaluated using the Hot Disk method: the XPS foam $\left(0.0308 \mathrm{~W} \mathrm{~m}^{-1} \mathrm{~K}^{-1}\right)$ and the SA superinsulator $(0.0141 \mathrm{~W}$ $\mathrm{m}^{-1} \mathrm{~K}^{-1}$ ). The study consisted into the overall comparison of the thermal conductivity of materials, always using the same probe $(7577, r=2.001 \mathrm{~mm})$. The power and time of recording were manually optimized in the $10-40 \mathrm{~s}$ and $3-7 \mathrm{~mW}$ ranges, respectively. The optimization consisted in selecting the conditions (power, time and number of data points) allowing reaching parameters (penetration depth, characteristic time) that match the values and ranges recommended by the Hot Disk manual. The results of such measurements are given Table 2 .

Table 2: Measurement results with the Hot Disk method.

\begin{tabular}{|c|c|c|c|c|}
\hline Material & $\begin{array}{c}\text { Thickness } t \\
(\mathrm{~mm})\end{array}$ & $\begin{array}{l}\lambda_{\text {estimated }} \\
\left(W m^{-1} K^{-1}\right)\end{array}$ & $\begin{array}{c}\lambda_{\text {ref }} \\
\left(W m^{-1} K^{-1}\right)\end{array}$ & $\begin{array}{c}\text { Deviation } \\
(\%)\end{array}$ \\
\hline \multirow{4}{*}{ XPS foam } & 3.03 & 0.0291 & \multirow{4}{*}{0.0308} & 5.52 \\
\hline & 4.46 & 0.0305 & & 0.97 \\
\hline & 6.00 & 0.0304 & & 1.30 \\
\hline & 9.00 & 0.0318 & & 3.25 \\
\hline \multirow{2}{*}{ SA } & 2.90 & 0.0264 & \multirow{2}{*}{0.0141} & 87.20 \\
\hline & 4.94 & 0.0264 & & 87.20 \\
\hline
\end{tabular}


The thermal conductivity of the XPS foam was correctly evaluated by the Hot Disk method (deviation lower than $6 \%$ ), but that of the super-insulating material SA was significantly overestimated (up to $87 \%$ ). The suspected reason of such discrepancy is heat transfer with air occurring at the connections of the probe wires (outside the sandwich). Indeed, this phenomenon is suspected to induce heat losses from the probe-sample sandwich, leading to an overestimation of the thermal conductivity. It should be noted that the deviation would have been far smaller with a hot disk of higher diameter ( $r=2.001 \mathrm{~mm}$ in our measurements).

\subsection{Application of the Calibrated Tiny Hot Plate (CTHP)}

The calibration was carried out based on the 4 pairs of XPS foam samples having the thicknesses and the thermal conductivity already reported in Table 1 . The current flowing throughout the heating element was adjusted for obtaining a difference of temperature between the opposite sides of the samples close to $10^{\circ} \mathrm{C}$. The corresponding data are gathered in Table 3.

Table 3. Experimental values of intensity and temperatures obtained for the XPS foam samples.

\begin{tabular}{|c|c|c|c|c|c|}
\hline Thickness $t$ & $T_{1}$ & $T_{0}$ & $T_{2}$ & $T_{\text {ext }}$ & Current \\
\hline$(\mathrm{mm})$ & \multicolumn{5}{|c|}{$\left({ }^{\circ} \mathrm{C}\right)$} \\
\hline 3.03 & 20.72 & 29.96 & 20.41 & 20.28 & 62.75 \\
\hline 4.46 & 20.71 & 30.12 & 20.43 & 20.37 & 57.80 \\
\hline 6.00 & 20.61 & 29.92 & 20.34 & 20.26 & 54.75 \\
\hline 9.00 & 20.73 & 30.09 & 20.43 & 20.4 & 52.76 \\
\hline
\end{tabular}

For various values of the exchange coefficient $h$ ranging from 4 to $7 \mathrm{~W} \mathrm{~m}^{-2} \mathrm{~K}^{-1}$, the values of $k$ leading to an estimated thermal conductivity equal to the one measured with the centered permanent hot plate (considered as a reference, see again Table 1) were determined for the sample of thickness $t=3.03 \mathrm{~mm}$. Those pairs of values $(h, k)$ were then used to estimate the thermal conductivity for the other sample thicknesses. The results thus obtained are presented in Fig. 5.

Based on these results, the values $h=5.2 \mathrm{~W} \mathrm{~m}^{-2} \mathrm{~K}^{-1}$ and $k=1.81 \times 10^{-3} \mathrm{~W} \mathrm{~K}^{-1}$ were selected as they allowed estimating the thermal conductivity of all samples with a relative difference lower than $0.1 \%$ with respect to the reference. Those values were then used for estimating the thermal conductivity of all the other samples listed in Table 1. Three measurements were carried out for each of the 7 pairs of samples, and the results are given in Table 4. It can be seen that the relative difference between the estimated values and the reference ones (centered hot plate) is always less than $7.2 \%$, and even close to $3 \%$ in most cases. The PVC is the exception, with a relative difference close to 9\%. Such larger discrepancy may be explained by the presence of contact resistances that are not totally negligible with respect to the thermal resistance $R_{\text {sample }}$. The thermal resistance $R_{\text {sample }}$ of all the samples are reported in Table 5. For PVC, $R_{\text {sample }}=2.9 \times 10^{-2} \mathrm{~K} \mathrm{~m}^{2} \mathrm{~W}^{-1}$ and the total thermal contact resistance can reach a maximum value $\frac{5 \times 10^{-5}}{0.025}=2.0 \times 10^{-3} \mathrm{~K} \mathrm{~m}^{2} \mathrm{~W}^{-1}$ if we consider an air layer with a thickness equal to the 
thermocouple diameter. In this case, the contact resistances are no longer totally negligible and thus lead to the underestimation of the thermal conductivity $\lambda$.

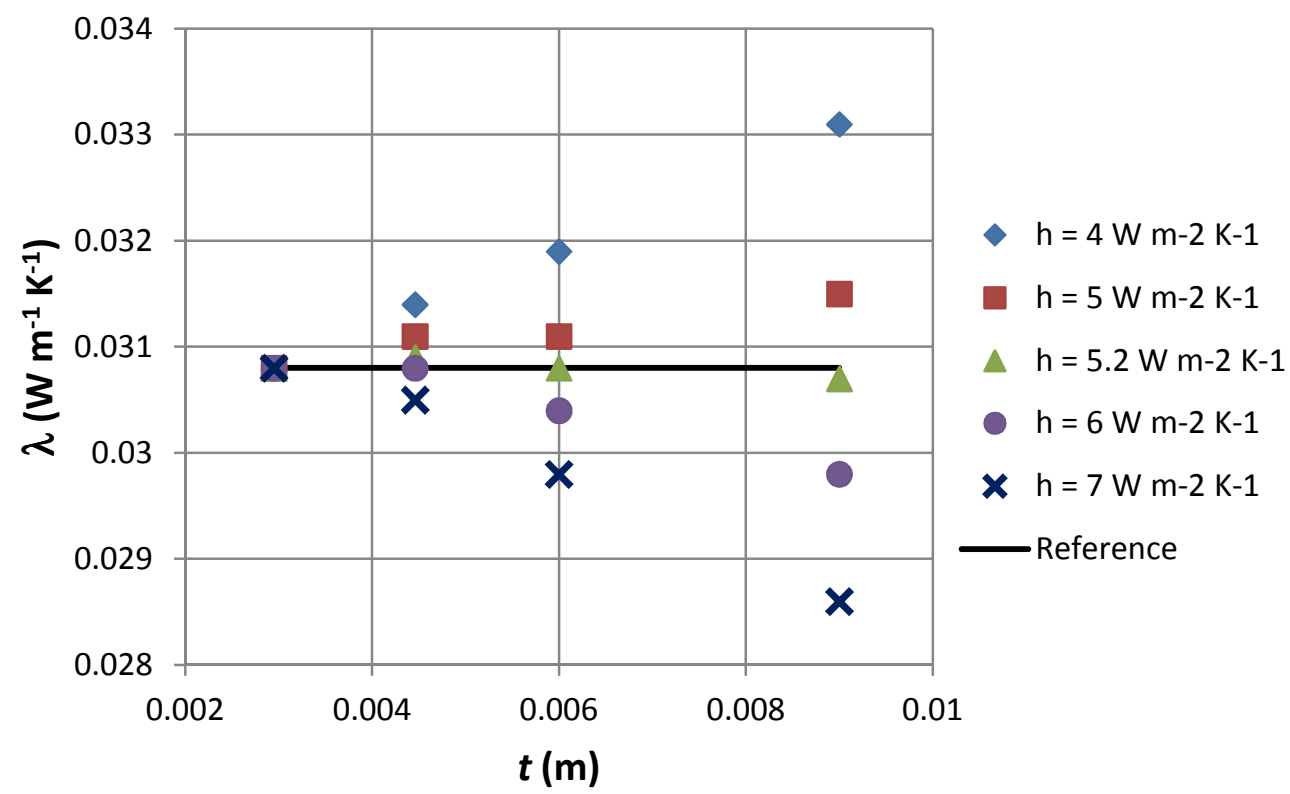

Figure 5. Estimated thermal conductivity of the XPS foam samples, $\lambda$, using various values of the exchange coefficient, $h$.

Table 4. Estimated thermal conductivity after 3 measurements on each of the other materials, and comparison with the results from the centered permanent hot plate method (reference).

\begin{tabular}{|c|c|c|c|c|c|c|c|c|c|}
\hline \multirow[t]{2}{*}{ Sample } & $\begin{array}{c}\text { Thickness } \\
t\end{array}$ & $\lambda_{1}$ & $\lambda_{2}$ & $\lambda_{3}$ & $\lambda_{\text {mean }}$ & $\begin{array}{l}\text { Standard } \\
\text { deviation }\end{array}$ & \multirow{2}{*}{$\begin{array}{c}\lambda_{\text {ref }} \\
\left(\mathrm{W} \mathrm{m}^{-1} \mathrm{~K}^{-1}\right)\end{array}$} & $\begin{array}{c}\text { Relative } \\
\text { difference } \\
\lambda_{\text {mean }} \text { vs. } \lambda_{\text {ref }}\end{array}$ & $\begin{array}{c}\lambda_{\text {mean }} \text { if } \\
\varphi_{\text {wires }}=0 \\
(k=0)\end{array}$ \\
\hline & $(\mathrm{mm})$ & \multicolumn{4}{|c|}{$\left(\mathrm{W} \mathrm{m}^{-1} \mathrm{~K}^{-1}\right)$} & $(\%)$ & & $(\%)$ & $\left(\mathrm{W} \mathrm{m}^{-1} \mathrm{~K}^{-1}\right)$ \\
\hline \multirow{3}{*}{ EPS foam } & 2.95 & 0.032 & 0.0313 & 0.0311 & 0.0315 & 1.5 & \multirow{3}{*}{0.0324} & -2.9 & 0.0468 \\
\hline & 5.80 & 0.0336 & 0.0332 & 0.0323 & 0.0330 & 2.1 & & 2.0 & 0.0623 \\
\hline & 8.74 & 0.0353 & 0.0359 & 0.033 & 0.0347 & 4.7 & & 7.2 & 0.0760 \\
\hline \multirow{2}{*}{$\begin{array}{c}\text { SA } \\
\text { superinsulator }\end{array}$} & 2.90 & 0.0146 & 0.0147 & 0.0152 & 0.0148 & 2.3 & \multirow{2}{*}{0.0141} & 5.2 & 0.0298 \\
\hline & 4.94 & 0.014 & 0.0146 & 0.0149 & 0.0145 & 3.3 & & 2.8 & 0.0385 \\
\hline PE foam & 6.30 & 0.0414 & 0.0424 & 0.0411 & 0.0416 & 1.7 & 0.0405 & 2.8 & 0.0735 \\
\hline PVC & 5.15 & 0.177 & 0.175 & 0.178 & \begin{tabular}{|l|}
0.177 \\
\end{tabular} & 0.8 & 0.193 & -8.5 & 0.0203 \\
\hline
\end{tabular}

Table 5: Thermal resistance of the samples

\begin{tabular}{|l|c|c|c|c|c|c|c|c|}
\hline Sample & & \multicolumn{3}{|c|}{ EPS foam } & SA superinsulator & PE foam & PVC \\
\hline Thickness & $\mathrm{mm}$ & 2.95 & 5.8 & 8.74 & 2.9 & 4.94 & 6.3 & 5.15 \\
\hline $\begin{array}{l}\text { Thermal } \\
\text { resistance }\end{array}$ & $\mathrm{K} \mathrm{m}^{-2} \mathrm{~W}^{-1}$ & $9.4 \times 10^{-2}$ & $1.8 \times 10^{-1}$ & $2.5 \times 10^{-1}$ & $2.0 \times 10^{-1}$ & $3.4 \times 10^{-1}$ & $1.5 \times 10^{-1}$ & $2.9 \times 10^{-2}$ \\
\hline
\end{tabular}


The average thermal conductivity which would have been estimated if the heat losses in the wires were negligible (i.e., $k=0$ ) is reported in the last column of Table 4. It can be seen that the error can be higher than $100 \%$ for the samples presenting the highest thermal resistances. The lower the thermal resistance, the lower the corresponding error because the heat flux that passes through the samples increases, and hence the proportion of the losses decreases. This is especially the case of the PVC sample.

The significant impact of the heat losses through both the wires feeding the heating element and the thermocouple wires was also evidenced as follows. For each experiment, the ratio of the heat flow rate lost through the wires to the total heat flow rate produced by the heater, $\varphi_{\text {wires }} / R I^{2}$, was calculated and the results are presented in Fig. 6 . It can be observed that the part of the total heat flow rate lost by the wires increases with the thermal resistance of the samples on the one hand, and that this ratio is higher than $30 \%$ for the most insulating samples on the other hand. This ratio is still higher than $10 \%$ for the PVC sample of thickness $5.15 \mathrm{~mm}$, hence it is never negligible. Therefore, any estimation that would not take the heat losses through the wires into account would thus be erroneous. It is worth mentioning, however, that the lower is the area of the heating element, the higher is the ratio of the losses to the total heat flow rate. The wires losses might thus become negligible only if the area of the heater (and hence of the sample) was really much higher.

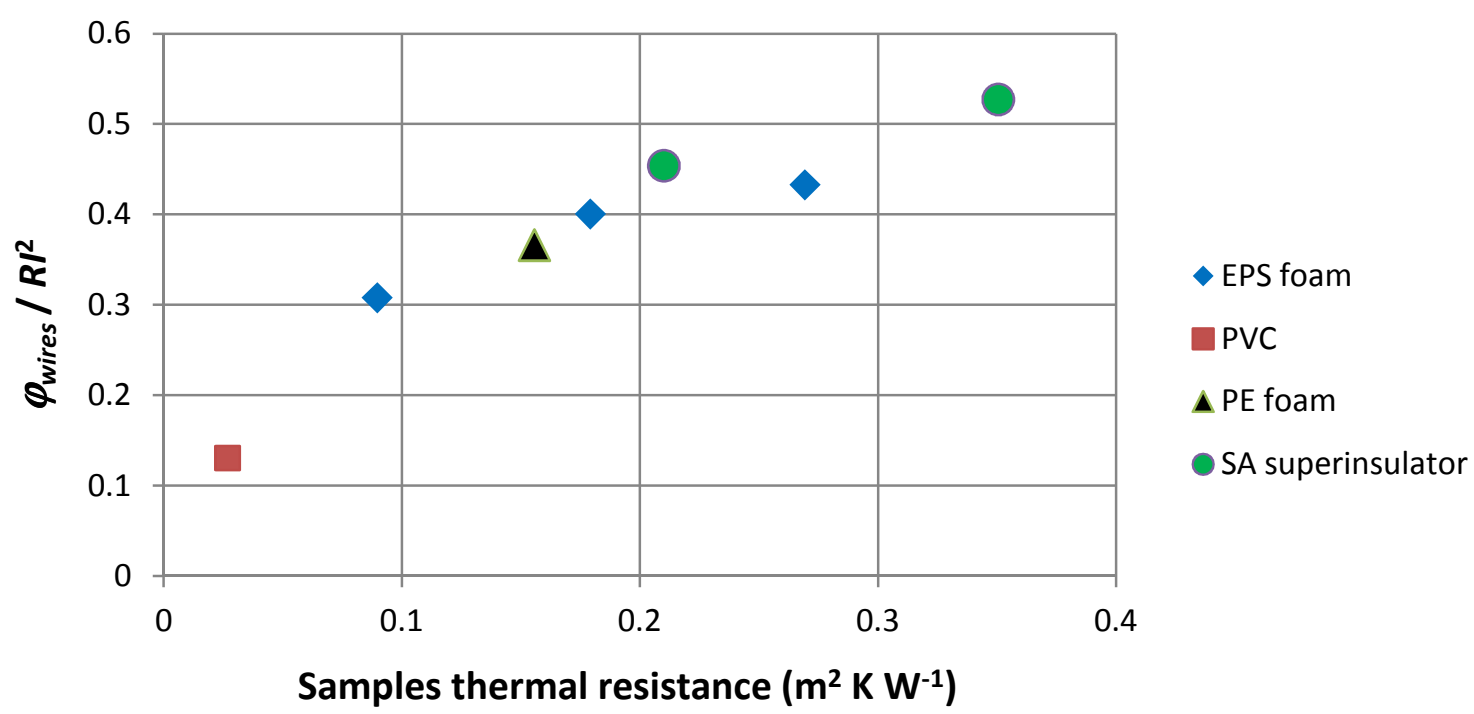

Figure 6. Ratio of the heat flow rate lost through the wires to the conduction heat flow rate, $\varphi_{\text {wires }} / R I^{2}$, as a function of the samples thermal resistance.

\section{Conclusion}

In the present study, a new method called "Calibrated Tiny Hot Plate" (CTHP) was presented for measuring accurately the thermal conductivity of small samples belonging to the families of traditional insulating to superinsulating materials. Developing such method appeared necessary after observing severe discrepancies between results from guarded hot plate and Hot Disk techniques. The Hot Disk may indeed be preferred not only because it is fast but mainly because it allows in practice the use of small samples. However, the experiments 
showed the impossibility of recovering the expected values of thermal conductivity of insulators that had been precisely characterized with a reference method.

The present work indeed stressed the dramatic impact of the heat lost through the wires of the heating element and of the thermocouple, especially as soon as such heater presents a low area: the smaller the heater (and hence the corresponding sample), the higher the error on the estimated value of thermal conductivity. The error was even higher, near $90 \%$, in the case of superinsulating material. Such problems were indisputably evidenced in the case of the Hot Disk method, whose relevance to the characterization of small and poorly conducting materials must therefore be questioned.

The new CTHP method was developed based on a metallized heating element sandwiched between two small cylindrical samples, and between which a thermocouple was inserted. A model of heat transfer in such system, combined with a calibration using a series of materials of various but accurately known thermal conductivities and thicknesses, allowed calculating a global heat exchange coefficient and another coefficient characterizing the losses throughout the wires (heater and thermocouple). The corresponding values finally allowed estimating thermal conductivities within the range $0.014-0.2 \mathrm{~W} \mathrm{~m}^{-1} \mathrm{~K}^{-1}$ based on samples having thicknesses from 3 to $9 \mathrm{~mm}$ and diameters as low as $15 \mathrm{~mm}$. The accuracy of this new method was much better than $10 \%$, which is a remarkable result for characterizing so small insulating materials.

\section{Acknowledgements}

The authors gratefully acknowledge the financial support of the CPER 2007-2013 "Structuration du Pôle de Compétitivité Fibres Grand'Est" (Competitiveness Fibre Cluster, France), through local (Conseil Général des Vosges), regional (Région Lorraine), national (DRRT and FNADT) and European (FEDER, France) funds. Dr. S. Schaefer acknowledges the support of the French Agency for the Environment and Energy Control (ADEME) through the "PANTHER" project for financing his postdoctoral contract.

\section{Acronyms and abbreviations}

CTHP : calibrated tiny hot plate (device or method)

EPS: expanded polystyrene (referring to the nature of EPS foam)

PE: polyethylene (referring to the nature of PE foam)

PVC: polyvinyl chloride (referring to the nature of PVC material)

XPS: extruded polystyrene (referring to the nature of opacified XPS foam)

SA: silica aerogel (referring to the nature of SA superinsulating material)

\section{Nomenclature}

D diameter of the sample

$h \quad$ convective and radiative loss coefficient

$\mathrm{mm}$

I current intensity

$\mathrm{W} \mathrm{m}^{-2} \mathrm{~K}^{-1}$

$J_{i} \quad$ Bessel function of the $\mathrm{i}^{\text {th }}$ order

$k \quad$ coefficient characterizing the losses throughout the wires

$\mathrm{mA}$

$\mathrm{W} \mathrm{K}^{-1}$ 


\begin{tabular}{|c|c|c|}
\hline$M$ & number of measurements & \\
\hline$N_{n}$ & quantity defined by eq. (4) & \\
\hline$r$ & radial coordinates or radius & $\mathrm{mm}$ \\
\hline$R$ & radius of the sample & $\mathrm{m}$ \\
\hline$R_{e l}$ & electrical resistance of the heating element & $\Omega$ \\
\hline$R_{\text {sample }}$ & thermal resistance of the sample & $\mathrm{K} \mathrm{m}^{2} \mathrm{~W}^{-1}$ \\
\hline$t$ & thickness of the sample & $\mathrm{mm}$ \\
\hline$T_{\text {ext }}$ & temperature of the thermally regulated enclosure & ${ }^{\circ} \mathrm{C}$ \\
\hline$T_{i}$ & temperature of bottom $(\mathrm{i}=1)$ or top $(\mathrm{i}=2)$ aluminum blocks & ${ }^{\circ} \mathrm{C}$ \\
\hline$T_{0}$ & surface temperature of the heating element & ${ }^{\circ} \mathrm{C}$ \\
\hline$Z$ & vertical axis coordinates & $\mathrm{mm}$ \\
\hline$\Delta P$ & penetration depth in Hot Disk measurement & $\mathrm{mm}$ \\
\hline$\lambda$ & thermal conductivity of the material & $\mathrm{W} \mathrm{m} \mathrm{m}^{-1} \mathrm{~K}^{-1}$ \\
\hline$\lambda_{\text {est }}$ & estimated thermal conductivity & $\mathrm{W} \mathrm{m} \mathrm{m}^{-1} \mathrm{~K}^{-1}$ \\
\hline$\lambda_{i}$ : & thermal conductivity estimated for the $\mathrm{i}^{\text {th }}$ measurement & $\mathrm{W} \mathrm{m} \mathrm{m}^{-1} \mathrm{~K}^{-1}$ \\
\hline$\lambda_{r e f}$ & reference thermal conductivity & $\mathrm{W} \mathrm{m}{ }^{-1} \mathrm{~K}^{-1}$ \\
\hline$\theta_{i}$ & difference of temperature between $T_{i}$ and $T_{\text {ext }}$ & ${ }^{\circ} \mathrm{C}$ \\
\hline $\bar{\theta}_{i}$ & local and z-dependent differential temperature, defined in eq. (3) & ${ }^{\circ} \mathrm{C}$ \\
\hline$\phi$ & Heat flux & $\mathrm{W} \mathrm{m}^{-2}$ \\
\hline$\varphi_{i}$ & sum of radial and vertical heat flow rates ( $\mathrm{i}=1$ : bottom, $\mathrm{i}=2$ : top) & $\mathrm{W}$ \\
\hline$\varphi_{r i}$ & radial heat flow rate leaving the samples sides ( $\mathrm{i}=1$ : bottom, $\mathrm{i}=2$ : top) & W \\
\hline$\varphi_{\text {wires }}$ & heat flow rate lost through the wires (heater and thermocouple) & W \\
\hline$\varphi_{z i}$ & vertical heat flow rate leaving the sample ( $\mathrm{i}=1$ : bottom, $\mathrm{i}=2$ : top) & $\mathrm{W}$ \\
\hline$\omega_{n}$ & solution of eq. (12) & \\
\hline
\end{tabular}

\section{References}

${ }^{1}$ Woodside W., "Deviation from one-dimensional heat flow in guarded hot plate measurements", Review of Scientific Instruments, 28, (1957)

${ }^{2}$ Rennex B., "Error Analysis for the National Bureau of Standards $1016 \mathrm{~mm}$ Guarded Hot Plate", Journal of Building Physics, 7 (1), (1983)

${ }^{3}$ Hammerschmidt U., "Guarded Hot-Plate (GHP) Method: Uncertainty Assessment", International Journal of Thermophysics, 23 ( 6), (2002)

${ }^{4}$ ISO 8302:1991 , "Thermal insulation -- Determination of steady-state thermal resistance and related properties -- Guarded hot plate apparatus", (1991)

${ }^{5}$ Aegerter M.A., Leventis N., Koebel M.M. (eds), “Aerogels handbook”, Springer, New York, 2011.

${ }^{6}$ Hrubesh L.W., Pekala R.W., "Thermal properties of organic and inorganic aerogels”, Journal of Material Research, 9(3), 731-738, (1994).

${ }^{7}$ Lu X., Caps R., Fricke J., Alviso C.T., Pekala R.W., "Correlation between structure and thermal conductivity of organic aerogels", Journal of Non-Crystalline Solids, 188, 226-234, (1995). 
${ }^{8}$ Gibson L.J., Ashby M.F., "Cellular Solids, structure and properties $-2^{\text {nd }}$ edition”, Cambridge University Press, Cambridge (UK), 2014.

${ }^{9}$ Delgado-Sánchez C., Santiago-Medina F., Fierro V., Pizzi A., Celzard A., "Optimisation of "green" tannin-furanic foams for thermal insulation by experimental design". Materials and Design, 139, 7-15, (2018)

${ }^{10}$ Degiovanni A. and Laurent A., "Une nouvelle technique d'identification de la diffusivité thermique pour la méthode flash", Revue de Physique Appliquée, 21, 229-237, (1986)

${ }^{11}$ Parker W.J., Jenkins R.J. and Butler C.P., "Flash method of determining thermal diffusivity, heat capacity and thermal conductivity", Journal of Applied Physics, 32 (9), 1679-1684, (1961)

${ }^{12}$ Gustafsson S.E., "Transient hot strip techniques for measuring thermal conductivity and thermal diffusivity", The Rigaku Journal, 4 (1-2), 16-28, (1987)

${ }^{13}$ Gobbé C., Iserna S., Ladevie B., "Hot strip method: application to thermal characterisation of orthotropic media”, International Journal of Thermal Sciences, 23 (10), 951-958, (2004).

${ }^{14}$ Gustavsson M., Karawacki E. and Gustafsson S.E., "Thermal conductivity, thermal diffusivity and specific heat of thin samples from transient measurement with Hot Disk sensors", Review of Scientific Instruments, 65, (1994)

${ }^{15}$ Bohac V., Gustavsson M.K., Kubicar L. and Gustafsson S.E., "Parameter estimations for measurements of thermal transport properties with the hot disk thermal constants analyzer", Review of Scientific Instruments, 71, 2452-2455, (2000)

${ }^{16}$ Jannot Y., Degiovanni A., "Thermal properties measurement of materials", ISTE Wiley editions, ISBN 978-1-78630-255-7, (2018)

${ }^{17}$ Jannot Y, Felix V, Degiovanni A, "A centered hot plate method for measurement of thermal properties of thin insulating materials", Measurement Science and Technology, 21, (2010)

${ }^{18}$ Jannot Y., Degiovanni A., Grigorova-Moutiers V., Godefroy J., "A passive guard for low thermal conductivity measurement of small samples by the hot plate method", Measurement Science and Technology, 28, 015008, (2017)

${ }^{19}$ Jannot Y., Rémy B., Degiovanni A., "Measurement of Thermal Conductivity and Contact Resistance Through a Tiny Hot-Plate Experiment", High Temperatures High Pressures, 39 (1), (2010). 